\title{
REDUCTION OF VIBRATIONS DUE TO DYNAMIC STALL IN HELICOPTERS USING AN ACTIVELY CONTROLLED FLAP
}

\author{
G. Depailler*and P. P. Friedmann ${ }^{\dagger}$ \\ Department of Aerospace Engineering \\ University of Michigan \\ Ann Arbor, Michigan 48109-2140
}

\begin{abstract}
$\underline{\text { ABSTRACT }}$
This paper presents a successful treatment of the helicopter vibration reduction problem at high advance ratios, taking into account the effects of dynamic stall. The ONERA model is used to describe the loads during stall, in conjunction with a rational function approximation for unsteady loads for attached flow. Single and dual actively controlled flaps are used to reduce vibrations. Several control laws are considered in this study. Successful vibration reduction is demonstrated over the entire range of advance ratios considered $(0.3 \leq \mu \leq 0.45)$. This study represents the first successful implementation of vibration reduction in presence of dynamic stall, and physical explanation for the vibration reduction process is also provided. Finally, saturation limits on the control deflections are imposed, which keep flap deflections in a practical range. Effective vibration reduction is achieved even when imposing practical saturation limits on the controller.
\end{abstract}

\section{NOMENCLATURE}

$a, a_{0}, a_{2}$

Separated flow empirical coefficients

$b \quad$ Blade semi chord

$\mathbf{C}_{\mathbf{0}}, \mathbf{C}_{\mathbf{1}}, \mathbf{D}, \mathbf{E}, \mathbf{R}$ Coefficient matrices from the RFA model

$C_{d 0} \quad$ Blade drag coefficient in attached flow

$C_{L}, C_{M}, C_{D} \quad$ Sectional lift, moment, drag coefficients

$c_{b} \quad$ Blade chord

*Ph. D. Candidate

$\dagger$ François-Xavier Bagnoud Professor, Fellow AIAA. Copyright (C)2001 by G. Depailler and P. P. Friedmann. Published by the American Institute of Aeronautics and Astronautics, Inc. with permission.

\begin{tabular}{|c|c|}
\hline$c_{c}$ & Flap chord \\
\hline$c_{w u}$ & Multiplier for $W_{u}$ weighting matrix \\
\hline$D$ & Drag force per unit span \\
\hline$D_{0}, D_{1}$ & Generalized flap motions \\
\hline d & Generalized force vector \\
\hline$E, E_{2}$ & $\begin{array}{l}\text { Separated flow empirical coeffi- } \\
\text { cients }\end{array}$ \\
\hline $\begin{array}{c}F_{H X 4}, F_{H Y 4} \\
F_{H Z 4}\end{array}$ & Nondimensional 4/rev hub shears \\
\hline$h$ & $\begin{array}{l}\text { Plunge displacement at the elastic } \\
\text { axis }\end{array}$ \\
\hline $\mathbf{h}$ & Generalized motion vector \\
\hline$J$ & Objective function \\
\hline$J_{R}$ & $\begin{array}{l}\text { Sum of the squares of the trim } \\
\text { residuals }\end{array}$ \\
\hline$k$ & Reduced frequency $\left(=\frac{\Omega b}{V}\right)$ \\
\hline$L$ & Lift force per unit span \\
\hline$L_{b}$ & Blade length \\
\hline$L_{c}$ & Control surface length \\
\hline$M$ & Mach number \\
\hline$M_{A C}$ & Pitch moment per unit span \\
\hline $\begin{array}{c}M_{H X 4}, M_{H Y 4} \\
M_{H Z 4}\end{array}$ & $\begin{array}{l}\text { Nondimensional } 4 / \mathrm{rev} \text { hub mo- } \\
\text { ments }\end{array}$ \\
\hline$N_{b}$ & Number of blades \\
\hline$p_{0}, p_{1}, p_{c}, p_{h}$ & Functions of $M$ \\
\hline$r, r_{0}, r_{2}$ & $\begin{array}{l}\text { Separated flow empirical coeffi- } \\
\text { cients }\end{array}$ \\
\hline$s_{l}$ & $\begin{array}{l}\text { Function of } M \text { derived from flat } \\
\text { plate theory }\end{array}$ \\
\hline$s_{m}, s_{d}$ & Empirical functions of $M$ \\
\hline$t$ & Time \\
\hline$t_{0}$ & Time when $\alpha=\alpha_{c r}$ \\
\hline $\mathbf{T}$ & Transfer matrix \\
\hline $\mathbf{u}_{\mathbf{i}}$ & $\begin{array}{l}\text { amplitudes of control input har- } \\
\text { monics }\end{array}$ \\
\hline
\end{tabular}




\begin{tabular}{|c|c|}
\hline$U$ & $\begin{array}{l}\text { Air velocity relative to the blade } \\
\text { section value }\end{array}$ \\
\hline$W_{0}, W_{1}$ & Generalized airfoil motions \\
\hline $\mathbf{W}_{\mathbf{z}}, \mathbf{W}_{\mathbf{u}}$ & Weighting matrices \\
\hline $\mathrm{x}$ & $\begin{array}{l}\text { Aerodynamic attached flow state } \\
\text { vector }\end{array}$ \\
\hline$x_{c}$ & Control surface position \\
\hline$X_{F A}, Z_{F A}$ & $\begin{array}{l}\text { Longitudinal and vertical offsets } \\
\text { between rotor hub and helicopter } \\
\text { aerodynamic center }\end{array}$ \\
\hline$X_{F C}, Z_{F C}$ & $\begin{array}{l}\text { Longitudinal and vertical offsets } \\
\text { between rotor hub and helicopter } \\
\text { center of gravity }\end{array}$ \\
\hline$z_{\mathbf{i}}$ & $\begin{array}{l}\text { amplitudes of vibratory load har- } \\
\text { monics }\end{array}$ \\
\hline$\alpha$ & Blade angle of attack \\
\hline$\alpha_{c r}$ & $\begin{array}{l}\text { Critical angle of attack for dy- } \\
\text { namic stall onset }\end{array}$ \\
\hline$\alpha_{f}, \alpha_{s}$ & Functions of $M$ \\
\hline$\alpha_{R}$ & Rotor shaft angle \\
\hline$\gamma$ & Lock number \\
\hline$\Gamma_{1}, \Gamma_{2}$ & Aerodynamic separated flow states \\
\hline$\Delta C_{L}$ & Measure of stall \\
\hline$\Delta t$ & Stall time delay \\
\hline$\theta_{0}, \theta_{1 s}, \theta_{1 c}$ & Collective and cyclic pitch angles \\
\hline$\theta_{t}$ & Tail rotor constant pitch \\
\hline$\kappa_{l}$ & $\begin{array}{l}\text { Function of } M \text { derived from flat } \\
\text { plate theory }\end{array}$ \\
\hline$\kappa_{m}, \kappa_{d}, \lambda$ & Empirical functions of $M$ \\
\hline$\mu$ & Advance ratio \\
\hline$\rho$ & Air density \\
\hline$\sigma$ & Function of $M$ \\
\hline$\phi_{R}$ & Lateral roll angle \\
\hline$\psi$ & Azimuth angle \\
\hline$\Omega$ & Rotor angular velocity \\
\hline$\omega_{F 1}, \omega_{L 1}, \omega_{T 1}$ & $\begin{array}{l}\text { Rotating fundamental blade fre- } \\
\text { quencies in flap, lead-lag and tor- } \\
\text { sion, respectively, nondimensional- } \\
\text { ized with respect to } \Omega \text {. }\end{array}$ \\
\hline () & Derivatives with respect to time \\
\hline
\end{tabular}

\section{Subscripts}

A Attached flow

$j \quad$ Represents $l, m$ or $d$

$l \quad$ coefficient connected to lift

$m \quad$ coefficient connected to moment

d coefficient connected to drag
$S \quad$ Separated flow

\section{Superscripts}

1

2

Inboard flap (in dual flap configuration)

Outboard flap (in dual flap configuration)

\section{INTRODUCTION AND BACKGROUND}

One of the primary concerns in rotorcraft design is the issue of vibrations and its reduction. High levels of vibration may lead to passenger discomfort, fatigue of helicopter components and increased noise. These phenomena decrease rotorcraft performance and increase cost. Thus, the issues of vibration prediction and its reduction to the lowest possible levels are of primary importance to the helicopter designer.

The largest contributor to vibrations in a helicopter is the rotor. The rotor blades transfer vibratory loads from the hub to the fuselage at harmonics that are predominantly $N_{b} / \mathrm{rev}$. The first methods devised for vibration reduction were passive, and were based on vibration absorbers and isolators. Later, active nethods have been implemented. In recent years, actively controlled trailing edge flaps have been investigated as a means for vibration control in helicopter rotors [1-5]. Experimental results from wind tunnels using the ACF were also presented by Straub [6]. Other vibration reduction studies using the ACF were also conducted $[7,8]$. Additional information on vibration reduction using the ACF can be found in a recent survey paper [9].

Active control strategies have been developed that can reduce vibration levels well below those achieved through traditional passive methods such as dampers and mass tuning [1]. Among the active control approaches, two fundamentally different strategies have emerged: higher harmonic control (HHC) and individual blade control (IBC). Three approaches have been used for individual blade control: actuation at the blade root [1], the actively controlled flap (ACF) [2-4], and active twist rotor blades $[10,11]$. Vibrations are controlled at their source, on the rotor blades, by manipulating the unsteady aerodynamic loading in the rotating system.

Dynamic stall is a phenomenon that affects helicopter performance at high advance ratios, and the vibrations induced by dynamic stall limit helicopter performance at high speeds. A good description of 
the dynamic stall phenomenon is provided in Chapter 9 of [12]. The main effects of dynamic stall are : (1) a a hysteretic dynamic lift coefficient that is much higher than the corresponding static value, accompanied by (2) large pitching moments; and (3) large increases in the pitch-link vibratory loads that manifest themselves in the pilot's stick and negatively affect controllability. The specific problems of reducing vibrations due to dynamic stall has been studied by Nguyen [13] using HHC, and only a very small amount of vibration reduction was achieved.

Among the available models [12] of dynamic stall, two semi-empirical models have become quite popular and are often used for computational modeling of rotorcraft vibration. These are the ONERA model [14], later modified by Truong [15] and the Leishman-Beddoes model [16].

Recently, Myrtle and Friedmann [3] developed a new compressible unsteady aerodynamic model for the analysis of a rotor blade with actively controlled flaps. This model is based on rational function approximation (RFA) of aerodynamic loads, and it has been shown that it produces good accuracy in aeroelastic simulations. De Terlizzi and Friedmann [4] included a nonuniform inflow distribution calculation, based on a free-wake model, in the analysis, and simulated vibration reduction at high speeds as well as alleviation of blade vortex interaction (BVI) at low advance ratios.

Valuable experimental results on the practical implementation of the ACF and its application to vibration reduction in the open loop mode, on a Machscaled two bladed rotor, were obtained by Fulton and Ormiston [17]. These results were compared with the simulation described in Refs. 4 and 18 and the correlation with the experimental data was found to be quite good, in most cases.

This paper has several objectives: (1) Development of an improved rotor aerodynamic model by incorporating dynamic stall in the aeroelastic simulation of rotor vibratory loads in forward flight; (2) Application of the simulation capability to the vibration reduction problem; and (3) Development of improved control laws for the vibration reduction problem. This paper represents an important contribution toward the improved fundamental understanding of vibration modeling and its reduction using the ACF under dynamic stall conditions.

\section{AEROELASTIC RESPONSE MODEL}

\section{Structural Dynamic Model}

The structural dynamic model is directly taken from [2]. The rotor is assumed to be composed of four identical blades, connected to a fixed hub, and it is operating at a constant angular velocity $\Omega$. The hingeless blade is modeled by an elastic beam cantilevered at an offset $e$ from the axis of rotation, as shown in Figure 1. The blade has fully coupled flap, lead-lag, and torsional dynamics. The strains within the blade are assumed to be small and the deflections to be moderate. The inertia loads are obtained from D'Alembert's principle and an ordering scheme is used to simplify the equations.

The control surfaces are assumed to be an integral part of the blade, attached at a number of spanwise stations. It is assumed that the control surfaces do not modify the structural properties of the blade, only the inertia and aerodynamic loads due to the flaps are accounted for. The control surface is constrained to pure rotation in the plane of the blade cross-section (see Fig. 1).

\section{Aerodynamic Model}

Aerodynamic Model For Attached Flow. Blade section aerodynamic loads are calculated using RFA, an approach described by Myrtle and Friedmann [3]. The RFA approach is an unsteady time-domain aerodynamic theory that accounts for compressibility, variations in the incoming flow and a combined blade, trailing edge flap configuration in the cross-section. These attributes make the RFA model particularly useful when studying vibration reduction in the presence of dynamic stall. The RFA approach generates approximate transfer functions between the generalized motion vector and the generalized attached flow force vector, as shown below:

$$
\begin{aligned}
\dot{\mathbf{x}}(t)= & \frac{U(t)}{b} \mathbf{R}(M) \mathbf{x}(t)+\mathbf{E}(M) \dot{\mathbf{h}}(t), \\
\mathbf{f}_{\mathbf{A}}(t)= & \frac{1}{U(t)}\left(\mathbf{C}_{0}(M) \mathbf{h}(t)+\mathbf{C}_{1}(M) \frac{b}{U(t)} \dot{\mathbf{h}}(t)\right. \\
& +\mathbf{D}(M) \mathbf{x}(t)) .
\end{aligned}
$$

A non-uniform inflow distribution, obtained from a free wake model is employed. The free wake model has been extracted [18] from the rotorcraft analysis tool CAMRAD/JA [19]. The wake vorticity is created in the flow field as the blade rotates, and 
then convected with the local velocity of the fluid. The local velocity of the fluid consists of the free stream velocity, and the wake self-induced velocity. The wake geometry calculation proceeds as follows: (1) the position of the blade generating the wake element is calculated, this is the point at which the wake vorticity is created; (2) the undistorted wake geometry is computed as wake elements are convected downstream from the rotor by the free stream velocity; (3) distortion of wake due to the wake self-induced velocity is computed and added to the undistorted geometry, to obtain a free wake geometry. The wake calculation model [19] is based on a vortex-lattice approximation for the wake.

\section{Aerodynamic Model For Separated Flow .} Two families of semi-empirical models that are extensively used and reasonably well documented are available. These are the ONERA family of models and the Leishman-Beddoes model. Both are computationally efficient and thus are suitable for modeling aerodynamic responses in comprehensive rotor analyses.

The ONERA Models. In this paper, the ONERA model as modified and presented by Petot [14] is used. This model was modified by Truong, using the mathematical concept of a Hopf bifurcation [15,20], but the resulting dynamic stall behavior displayed an oscillatory behavior which was not supported by experimental evidence. Useful modifications to the ONERA model were also introduced by Peters [21]. A brief description of the model as implemented in this paper is provided next. The airfoil velocity is expressed using the generalized motions $W_{0}$, $W_{1}$ shown in Fig. 2 and defined by:

$$
W_{0}=U \alpha+\dot{h}, \quad W_{1}=b \dot{\alpha}
$$

The model establishes a transfer function between the generalized motion vector $\mathbf{d}=\left[W_{0}, W_{1}, D_{0}, D_{1}\right]$ and the generalized force vector $\mathbf{h}=\left[L, M_{A C}, D\right]$. It is based on linear, time-varying coeffient differential equations. A first-order equation for attached flow

$$
\begin{aligned}
\dot{\Gamma_{1}}+\lambda \frac{U}{b} \Gamma_{1}= & \lambda \frac{U}{b} p_{0} W_{0}+\lambda \frac{U}{b} W_{1}+\alpha_{s} p_{0} \dot{W}_{0} \\
& +\alpha_{s} \sigma \dot{W}_{1},
\end{aligned}
$$

where $\lambda, \alpha_{s}, p_{0}, \sigma$ are functions of $M$ derived from flat plate theory, and three second-order ones for separated flow:

$$
\begin{aligned}
\ddot{\Gamma_{j 2}+a_{j} \cdot \frac{U}{b}} \dot{\Gamma_{j 2}}+r_{j}\left(\frac{U}{b}\right)^{2} \Gamma_{j 2}= & -\left[r_{j}\left(\frac{U}{b}\right)^{2} V \Delta C_{L}\right. \\
& +E_{j} \cdot \frac{U}{b} \dot{W}_{0},
\end{aligned}
$$

where $j=l, m, d$. The loads are derived from these expressions

$$
\begin{gathered}
L_{S}=\frac{1}{2} \rho c_{b}\left(s_{l} b \dot{W}_{0}+\kappa_{l} b \dot{W}_{1}+U\left(\Gamma_{l 1}+\Gamma_{l 2}\right)\right. \\
M_{A C_{S}}=\frac{1}{2} \rho c_{b}^{2}\left(s_{m} b \dot{W}_{0}+\kappa_{m} b \dot{W}_{1}+U\left(\Gamma_{m 1}+\Gamma_{m 2}\right)\right. \\
D_{S}=\frac{1}{2} \rho c_{b}\left(s_{d} b \dot{W}_{0}+\kappa_{d} b \dot{W}_{1}+U\left(\Gamma_{d 1}+\Gamma_{d 2}\right)\right.
\end{gathered}
$$

The attached flow loads in the ONERA model have been modified by Peters [21] to be consistent with Greenberg's unsteady aerodynamic theory. Other features of the ONERA dynamic stall model include the presence of a time delay for lift stall, expressed in non-dimensional time, and the presence of 18 empirical coefficients, 6 each $\left(r_{j 0}, r_{j 2}, a_{j 0}, a_{j 2}, E_{j 2}\right)$ associated with lift $(j=l)$, moment $(j=m)$, and drag $(j=d)$. The coefficients are

$$
\begin{gathered}
r_{j}=\left(r_{j 0}+r_{j 2} \cdot \Delta C_{L}^{2}\right)^{2} \\
a_{j}=a_{j 0}+a_{j 2} \cdot \Delta C_{L}^{2} \\
E_{j}=E_{j 2} \cdot \Delta C_{L}^{2}
\end{gathered}
$$

The quantity $\Delta C_{L}$ is called a measure of stall and can attain two possible values:

$$
\Delta C_{L}=0
$$

$$
\Delta C_{L}=\left(p_{0}-p_{1}\right)\left(\alpha-\alpha_{f}\right) p_{c}\left[e^{p_{h}\left(\alpha-\alpha_{c r}\right)}-1\right]
$$

The separation criterion is based on the angle of attack, and three possible cases can occur. Case 1: if $\alpha<\alpha_{c r}=15^{\circ}\left(1-M^{2}\right), \Delta C_{L}$ is given by Eq. (12). Case 2: assume that at time $t=t_{0}, \alpha=\alpha_{c r}, \dot{\alpha}>0$; then, at time $t>t_{0}+\Delta t, \Delta C_{L}$ is given by Eq. (13). As $\Delta C_{L}$ is different from zero, separated flow loads become substantial. Case 3: when $\alpha<\alpha_{c r}$, $\Delta C_{L}$ is set to zero again (Eq. 12) and the separated flow loads quickly decrease to zero. Attached and separated flow loads are then added at each blade section, i. e.:

$$
\begin{array}{r}
C_{L}=C_{L_{A}}+C_{L_{S}}, \quad C_{M}=C_{M_{A}}+C_{M_{S}}, \\
C_{D}=C_{d 0}+C_{D_{S}}
\end{array}
$$


Leishman-Beddoes Model. The LeishmanBeddoes model [16] is based on indicial response approach and the Duhamel superposition integral method. For lift and moment, noncirculatory loading comes from piston theory, while the circulatory part is similar to a Küssner-like function. Indicial responses are cast in first-order linear differential equation form. The trailing-edge separation is implemented using Kirchhoff flow. Under unsteady flow, the position of the separation point is modified using an effective angle of attack. The LeishmanBeddoes model has only four empirical constants. Thus, this model has the least number of empirical coefficients. Other coefficients are derived using thin-airfoil theory. Furthermore, constants change according to flight condition.

Brief Comparison of the Two Dynamic Stall Models. A very comprehensive study that compares several dynamic stall models, in their ability to predict rotor behavior in presence of dynamic stall has been completed recently by a European study group [22]. Here we conduct only a brief comparison of the two models mentioned earlier. The Leishman-Beddoes lift model is slightly superior to the ONERA model, especially at peak lift, but the difference between the models is small elsewhere. The moment downward peak is also underpredicted by the ONERA model. However, the ONERA model is much more suitable for combination with the RFA type unsteady aerodynamic model which accounts for unsteadiness, compressibility and presence of flap. Furthermore, the ONERA model uses the same generalized motion vector $\mathbf{h}=\left[\begin{array}{llll}W_{0}, & W_{1}, & D_{0}, & D_{1}\end{array}\right]$ for both regimes. In both RFA and the ONERA model, the attached flow transfer function is approximated by a rational transfer function. Both models are formulated in the time domain. Therefore, compatibility between attached flow and separated flow is not an issue.

Optimization of Aerodynamic Coefficients. The documentation in the literature on Petot's selection of empirical coefficientsis only partially complete. Furthermore, inaccuracies were noticed in the prediction of the pitching moment downward peak (Fig. 3). This peak, an important feature of dynamic stall, is underpredicted by $30 \%$, which requires a modification of the coefficients in the pitch equation. The ONERA model for lift and drag does not display any such inaccuracy, and their coefficients did not rquire a modification. The approach used for the selection of the coefficients used for the moment computation, under stalled conditions, is based on an optimization routine due to Powell [23] for curve-fitting. In this curve fitting the representation of the peak in the downward pitching moment coefficient is emphasized. The portion of the curve where $\alpha<12^{\circ}$ has not been weighted, since dynamic stall loads are very small for this case. Two flight conditions, one for light stall and one for medium-deep stall, are consdidered for curve-fitting: $\alpha \in\left[-5^{\circ}, 15^{\circ}\right]$ and $\alpha \in\left[0^{\circ}, 20^{\circ}\right]$. For both cases, the Mach number and reduced frequency were assumeed to be $M=0.3$ and $k=0.1$. The results for medium-deep stall are presented in Fig. 3. The downward peak in the pitching moment and the portion of the curve for $\alpha>15^{\circ}$ are reproduced in a more accurate manner, when using the optimized coefficients. In this case, the error in the peak magnitude error is then less than $5 \%$, and the new moment coefficients are:

$$
\begin{array}{r}
a_{m 0}=0.044, \quad a_{m 2}=0.13, \quad r_{m 0}=0.18 \\
r_{m 2}=0.39, \quad e_{m 2}=0.93
\end{array}
$$

Figure 4 shows the percentage of change in the vibratory loads when dynamic stall coefficients are changed by $10 \%$. All changes except one are less than $5 \%$ with the majority below $1 \%$. Thus, changes in these coefficients seem to have a minor influence on $4 /$ rev vibratory loads. It is also evident that the vertical hub shear, which plays an important role for vibration reduction, is sensitive on only one empirical coefficient, namely $r_{m 0}$. This coefficient [14] was only changed by a small amount, $10 \%$, from $r_{m 0}=0.20$ to $r_{m 0}=0.18$.

Combined Aerodynamic Model. The complete aerodynamic model used in this study consists of the RFA model for attached flow loads, using a free wake model in order to obtain the non-uniform inflow. The ONERA dynamic stall model is used for separated flow loads. Thus the complete aerodynamic state vector for each blade section consists of RFA attached flow states and ONERA separated flow states, together with the representation of the free wake.

\section{METHOD OF SOLUTION}

The blade is discretized [2] using the global Galerkin method, based upon the free vibration modes of the rotating blade. Three flapping modes, two lead-lag modes and two torsional modes are used in the actual implementation. The combined structural and aerodynamic equations form a system of coupled differential equations than can be 
cast in state variable form. They are then integrated in the time domain using the AdamsBashfort DE/STEP predictor-corrector algorithm. The trim procedure [18] enforces three force equilibrium equations (longitudinal, vertical and lateral forces) and three moment equilibrium equations (roll, pitch and yaw moments). A simplified tail rotor model is used, using uniform inflow and blade element theory. The six trim variables are the rotor shaft angle $\alpha_{R}$, the collective pitch $\theta_{0}$, the cyclic pitch $\theta_{1 s}$ and $\theta_{1 c}$, the tail rotor constant pitch $\theta_{t}$ and the lateral roll angle $\phi_{R}$. The trim procedure is based on the minimization of the sum $J_{R}$ of the squares of trim residuals. At high advance ratios $(0.30<\mu \leq 0.35)$ in the presence of dynamic stall, an autopilot procedure described in [24] is used to accelerate convergence to the trim state. At higher advance ratios $(0.35<\mu)$, an iterative optimization program based on Powell's method is used to find the trim variables that minimize $J_{R}$.

\section{CONTROL ALGORITHMS}

This section presents a brief description of the control strategies that are employed in this aeroelastic simulation study of vibration reduction. Two different implementations of active control configurations are studied: (a) a single, actively controlled partial span trailing edge flap; and (b) a dual flap configuration, shown in Fig. 5, in which each flap is independently controlled. In each case, the controller will act to reduce the $4 / \mathrm{rev}$ vibratory hub shears and moments.

The control strategy is based on the minimization of a performance index described in $[1-5,25]$ that is a quadratic function of the vibration magnitudes $\mathbf{z}_{i}$ and control input amplitudes $\mathbf{u}_{i}$ :

$$
J=\mathbf{z}_{i}^{T} \mathbf{W}_{\mathbf{z}} \mathbf{z}_{i}+\mathbf{u}_{i}^{T} \mathbf{W}_{\mathbf{u}} \mathbf{u}_{i}
$$

The subscript $i$ refers to the i-th control step, reflecting the discrete-time nature of the control. The time interval between each control step must be long enough to allow the system to return to the steady state so that the $4 / \mathrm{rev}$ vibratory magnitudes can be accurately measured. The matrices $\mathbf{W}_{\mathbf{z}}$ and $\mathbf{W}_{\mathbf{u}}$ are weighting matrices on the vibration magnitude and control input, respectively.

\section{Conventional Control Approach (CCA)}

A linear, quasistatic, frequency domain representation of the vibratory response to control inputs is used $[2,3,18]$. The input harmonics are related to the vibration magnitudes through a transfer matrix $\mathbf{T}$, given by

$$
\mathbf{T}=\frac{\partial \mathbf{z}_{\mathbf{i}}}{\partial \mathbf{u}_{\mathbf{i}}}
$$

The optimal control is:

$$
\mathbf{u}_{i}^{*}=-\mathbf{D}^{-1} \mathbf{T}^{T}\left\{\mathbf{W}_{\mathbf{z}} \mathbf{z}_{i-1}-\mathbf{W}_{\mathbf{z}} \mathbf{T} \mathbf{u}_{i-1}\right\}
$$

where

$$
\mathbf{D}=\mathbf{T}^{T} \mathbf{W}_{\mathbf{z}} \mathbf{T}+\mathbf{W}_{\mathbf{u}}
$$

\section{Algorithm Resembling Simulated Annealing}

A method that resembles simulated annealing was developed by Meerkov [26] to find the global minimum of the function $J$. This method, denoted as the simulated annealing algorithm (SAA) assumes the availability of an algorithm for obtaining a local minimum when starting from an initial guess, and it proceeds to establish an algorithm for finding the global minimum, in an effective manner. This approach is mathematically more refined than the CCA, and its potential for payoffs warranted its application to the vibration reduction problem.

\section{Control in the Presence of Flap Deflection \\ Saturation}

In the practical implementation of the $\mathrm{ACF}$, adaptive materials based actuation, using piezoelectric or magnetostrictive materials, has been extensively studied. Adaptive materials are limited in their force and stroke producing capability, leading to fairly small angular deflections. From a control perspective this leads to saturation which introduces serious problems for vibration control. This important problem was studied and solved effectively in a recent paper by Cribbs and Friedmann [27]. This approach to dealing with saturation, described below, is also used in this paper. Saturation is treated by the auto weight approach [27]. The weighting matrix $\mathbf{W}_{\mathbf{u}}$ is represented in a form which allows its modification by premultiplying it by a scalar $c_{w u}$ that is continuously adjusted. The controller manipulates the scalar multiplier to provide the proper flap constraints. If the flap deflection is overconstrained, the controller reduces the value of $c_{w u}$ and a new optimal control is calculated. If the flap deflection is underconstrained, the controller increases the value of $c_{w u}$ and a new optimal control is calculated. The iterative procedure reduces or increases $c_{w u}$ until the optimal control converges to the desired deflection limits within a prescribed tolerance. 
Table 1: Elastic blade configuration

\begin{tabular}{ll}
\hline Rotor Data & \\
$N_{b}=4$ & $c_{b}=0.05498 L_{b}$ \\
$\omega_{F 1}=1.123$ & $C_{d o}=0.01$ \\
$\omega_{L 1}=0.732$ & $C_{m o}=0.0$ \\
$\omega_{T 1}=3.17$ & $a_{o}=2 \pi$ \\
$\gamma=5.5$ & $\sigma=0.07$ \\
Helicopter Data & \\
$\bar{C} C_{W}=0.00515$ & \\
$X_{F A}=0.0$ & $Z_{F A}=0.3$ \\
$X_{F C}=0.0$ & $Z_{F C}=0.3$ \\
\hline
\end{tabular}

Table 2: Flap configurations

\begin{tabular}{ll}
\hline$c_{c}=0.25 c_{b}$ & \\
Single Flap & \\
\hline$x_{c}=0.75 L_{b}$ & $L_{c}=0.12 L_{b}$ \\
Dual Flap & \\
\hline$x_{c}^{1}=0.72 L_{b}$ & $L_{c}^{1}=0.06 L_{b}$ \\
$x_{c}^{2}=0.92 L_{b}$ & $L_{c}^{2}=0.06 L_{b}$ \\
\hline
\end{tabular}

\section{RESULTS}

The helicopter configuration used in this study resembles approximately a MBB BO-105 four-bladed hingeless rotor. The data used in the computations is summarized in Table 1. The characteristics of the single and dual flap configurations are shown on Table 2. The portion of the blade spanned by the single flap is equal to the sum of the span covered by the dual flap configuration (see Fig. 5).

The effect of dynamic stall on the trim condition is examined, first. The presence of dynamic stall does not significantly affect the collective and cyclic pitch angles. As evident from Fig. 6, except for $\mu=0.45$ where changes of the order of $10 \%$, which amounts to 1.4 degrees, are present. At an advance ratio $\mu=0.30$ and in the presence of dynamic stall, the standard autopilot trim convergence accelerator is not successful and therefore Peters' [24] method was used. At higher values of $\mu, \mu>0.35$, Powell's method [23] is used. The rotor shaft angle, shown in Fig. 7, is the only other trim variable that is significantly affected by dynamic stall. This may be due to the overestimation of sectional lift at high angles of attack when the RFA model is used. This linear model does not capture the post-stall nonlinear behavior of the lift coefficient.

Next, the effect of dynamic stall on the baseline 4/rev vibratory hub loads.is considered. Fig- ure 8 depicts the $4 /$ rev vibratory loads at $\mu=0.35$ when dynamic stall is included. All vibratory loads are increased significantly when dynamic stall is accounted for in the simulation. The pitching hub moment is increased by $50 \%$, the rolling hub moment by $60 \%$. The horizontal and lateral hub shears, as well as the yawing hub moment, are more than doubled. The most important vibratory component, the vertical hub shear, is increased by a factor of three. Therefore, the adverse effects of dynamic stall on vibratory hub loads are evident in the simulation.

Vibration reduction in the presence of dynamic stall, at high advance ratios, is considered next. For this case the vibration reduction capability of both single and dual flap configurations is considered. Figure 9 shows the vibration reduction capabilities of the two flap configurations. The single flap does not achieve reduction in vertical hub shear, but all other vibratory loads are reduced by $70-85 \%$. The dual flap configuration reduces all loads by 70-95\% and is at least $40 \%$ more effective than the single flap approach. This comparison shows the superiority of the dual flap configuration over the single flap. Excellent vibration reduction in presence of dynamic stall is achieved by this configuration. This reduction is much better than what has been documented in the literature before [13].

Figure 10 represents the baseline angle of attack distribution over the rotor disk, at $\mu=0.30$. As expected, over the retreating blade, angles of attack become large and exceed $\alpha_{c r}$ between $\psi=250^{\circ}$ and $\psi=300^{\circ}$. Figure 11 represents the angle of attack distribution when optimal single flap control is applied. The presence of control reduces the angle of attack of the blade by approximately $1^{\circ}$, over the whole surface of the rotor disk. This reduces the area affected by dynamic stall. Figure 12 depicts the dynamic stall locus, as defined by flow separation and reattachment, without control (diamonds) and with control (squares). The dynamic stall termination changes little in the presence of control (the difference in azimuth does not exceed $2^{\circ}$ ), however the onset of dynamic stall has been significanlty altered. The boundaries of the dynamic stall zone is reduced by $30 \%$ from a region that extends between $240^{\circ} \leq \psi \leq 290^{\circ}$ to a region that is much narrower $255^{\circ} \leq \psi \leq 290^{\circ}$. This essentially explains the mechanism of vibration reduction by active control.

Figure 13 shows the optimal flap deflections required for the vibration reduction in the single flap configuration. The maximum flap amplitudes are about $15^{\circ}$. Figure 14 displays the flap deflections for the dual flap configuration; here again, the maximum deflection of both flaps is about $15^{\circ}$. How- 
ever, actuator technologies based on smart materials severely limit flap deflections to a maximum of $5^{\circ}$. Furthermore, flap deflections of $15^{\circ}$ are not acceptable from a practical implementation point of view. Therefore, additional results taking into account actuator saturation that allows practical limits on flap deflections have been obtained. The maximum allowable flap deflection for the cases considered here was set to $4^{\circ}$, which is the value considered in an earlier study [27]. Results for vibration reduction are presented in Fig. 15 for the single flap configuration. The vertical hub shear is unchanged, but vibratory hub shear reduction is not affected by saturation; reductions of $70-80 \%$ are obtained again. However, vibratory hub moments are reduced $60-85 \%$ instead of $80-90 \%$.

These results indicate that vibration reduction with the single flap configuration operating with and without saturation limits is similar, where the unsaturated flap reduces vibratory hub loads 10-30\% more than the saturated flaps. These results are consistent with the observation made in an earlier paper [27] where the effects of dynamic stall were not included.

Figure 16 shows the flap deflections with and without saturation for the single flap configuration. The maximum allowed flap deflections occurs at $\psi=225^{\circ}$, that is just before a large portion of the blade enters dynamic stall. This result confirms that the main feature of the control is to postpone dynamic stall entry (Figs. 10-11). When saturation is not taken into account, flap deflections are unconstrained, and large deflections can occur while producing only a small amount of vibration alleviation. This appears to be the case on the advancing portion part of the rotor disk. Figure 17 shows flap deflections for the inboard flap of the dual flap configuration. On the inboard flap, the saturation limit is never attained and the maximum flap deflection is less than $2^{\circ}$. This is a reflection upon the nonlinearity in the problem combined with the existence of multiple minima in the objective function $J$. The outboard flap deflections are displayed in Fig. 18. Again the maximum flap deflection is not attained. For both the inboard and outboard flaps, high amplitudes are evident, when saturation is included, in the range $180^{\circ}<\psi<270^{\circ}$, which corresponds to the onset of dynamic stall. This is consistent with our earlier remark that control tends to delay the onset of dynamic stall.

Figures 19-20 illustrate the effectiveness of vibration reduction when the CCA is replaced by a different algorithm which resembles simulated annealing [26]. The performance of the CCA and SAA algorithms for the single flap case are compared in Fig. 19. The most important component of the hub shears, the $4 / \mathrm{rev}$ vibratory hub shear, is reduced by $10 \%$ when compared to the CCA. The horizontal hub shear component in the lateral direction is decreased by an additional $50 \%$, and the hub rolling moment is also reduced by $50 \%$. On the other hand, the fore and aft component of the hub shear is slightly increased, and the yawing moment increases by $30 \%$. Clearly, the SAA control algorithm has only a slight advantage when compared with the CCA algorithm. There is a large amount of practical experience with the CCA in the rotary-wing vibration reduction field, and therefore the slight advantage of the SAA algorithm cannot justify its adoption. The maximum flap deflections required for the implementation of these two control algorithms are compared in Fig. 20, and there is no substantial difference between these two cases.

The vibration reduction results presented in the previous figures were at an advance ratio of $\mu=$ 0.35. However, it is well known that the unfavorable effects associated with dynamic stall increase rapidly with the advance ratio. This provides the justification for re-examining the results at a higher advance ratio, so as to identify possible problems that can emerge for these more severe flight conditions.

Vibration reduction results at the advance ratio $\mu=0.45$ using the single flap configuration are presented in Fig. 21. Using the CCA approach, the vibration reduction achieved is 20-25\% in longitudinal hub shear and yawing hub moment; $50 \%$ in lateral hub shear and rolling hub moment, $80 \%$ in pitching hub moment, however the vertical hub shear in increased by $80 \%$, which is unacceptable since it represents the most important component of the vibrations. When saturation is accounted for, all loads decrease, between $10 \%$ for longitudinal force and $60 \%$ for vertical force. Therefore, vibration reduction is satisfactory, when actuator saturation is considered. This interesting result could be due to large nonlinearities associated with dynamic stall which give rise to multiple minima of $J$. Only under these extreme flight conditions are controlled vibratory loads higher than their uncontrolled level at $\mu=0.30$.

\section{CONCLUSIONS}

A fairly extensive numerical simulation of vibration reduction at high speed flight using actively controlled flaps has been conducted. The ONERA 
dynamic stall model was used for the representation of the unsteady aerodynamic loading in the separated flow region. Both single flap and dual flap configurations were studied, and limits on flap deflections were imposed. The principal conclusions obtained are provided below.

1. The ACF implemented either as a single flap or in the dual flap configuration is an effective means for alleviating the unfavorable effects due to dynamic stall.

2. The physical mechanism for reducing vibrations due to dynamic stall appears to be associated with delayed entry of the retreating blade into the stall region; combined with a reduction in the stall region over the area of the disk

3. The CCA algorithm that has been used for the last two decades in the area of vibration reduction in rotorcraft appears to be an excellent algorithm. A more refined control algorithm such as the SAA does not have a clear cut advantage over CCA.

4. The dual flap configuration appears to have an advantage over the single flap configuration in its ability to alleviate the undesirable effects associated with dynamic stall.

5. The actively controlled flap, implemented in either single or dual flap configurations, is more effective at alleviating dynamic stall effects than the HHC approach studied in Ref. 13. The primary reason for the effectiveness of ACF is due to the fact that it represents a local controller, that is inherently more suitable for dealing with local effects such as dynamic stall. The $\mathrm{HHC}$ approach affects the entire blade and thus is at a disadvantage when attempting to alleviate local effects.

6. Imposition of flap deflection limits, and the appropriate treatment of saturation play an important role in the ability of the ACF, in both configurations, to achieve alleviation of dynamic stall related effects. Therefore, a careful treatment of these issues is necessary for the practical implementation of the ACF in rotorcraft.

\section{REFERENCES}

[1] Friedmann, P. P. and Millot, T. A., "Vibration Reduction in Rotorcraft Using Active Control: A Comparison of Various Approaches,"
Journal of Guidance, Control, and Dynamics, Vol. 18, No. 4, No. 4, 1995, pp. 664-673.

[2] Millott, T. A. and Friedmann, P. P., "Vibration Reduction in Helicopter Rotors Using an Actively Controlled Partial Span Trailing Edge Flap Located on the Blade," NASA, CR 4611, 1994.

[3] Myrtle, T. F. and Friedmann, P. P., "Application of a New Compressible Time Domain Aerodynamic Model to Vibration Reduction in Helicopters Using an Actively Controlled Flap," Journal of the American Helicopter Society, No. 1, No. 1, 2001, pp. 32-43.

[4] De Terlizzi, M. and Friedmann, P. P., "Active Control of BVI Induced Vibrations Using a Refined Aerodynamic Model and Experimental Correlation," 55th Annual Forum of the American Helicopter Society, Montréal, Canada, May 1999, pp. 599-618.

[5] Friedmann, P. P., de Terlizzi, M. and Myrtle, T. F., "New Developments in Vibration Reduction with Actively Controlled Trailing Edge Flaps," Mathematical and Computer Modeling, Vol. 33, 2001, pp. 1055-1083.

[6] Straub, F. K., "Active Flap Control for Vibration Reduction and Performance Improvement," Proceedings of the 51st American Helicopter Society Forum, Fort Worth, Tex., May 1995, pp. 381-392.

[7] Straub, F. K. and Charles, B. D., "Comprehensive Modeling of Rotors with Trailing Edge Flaps," Proceedings of the 55th Forum of the American Helicopter Society, Montréal, Canada, May 1999.

[8] Chopra, I.., Milgram, J. and Straub, F. K., "Comprehensive Rotorcraft Aeroelastic Analysis with Trailing Edge Flap Model," Proceedings of the 52th Forum of the American Helicopter Society, Washington, D. C., June 1996, pp. $715-725$.

[9] Friedmann, P. P., "Rotary-Wing Aeroelasticity - Current Status and Future Trends," AIAA Paper No. 2001-0427, Proceedings of the 39th AIAA Aerospace Sciences Meeting and Exhibit, Reno, Nev., January 2001.

[10] Shin, S. J. and Cesnik, C. E. S., "Forward Flight Response of the Active Twist Rotor for Helicopter Vibration Reduction," 
AIAA Paper No. 2001-1357, Proceedings of the 42th AIAA/ASME/ASCE/AHS/ACS Structures, Structural Dynamics and Materials Conference, Seattle, Wash., April 2001.

[11] Wilbur, M. L., Mirick P. H., Yeager, W. T., Langston, C. W., Shin, S. J. and Cesnik, C. E. S., "Vibratory Loads Reduction Testing of the NASA/Army/MIT Active Twist Rotor," Proceedings of the American Helicopter Society 57th Annual Forum, Washington, D. C., May 2001.

[12] Leishman, J. G., Principles of Helicopter Aerodynamics, Cambridge, Cambridge University Press, 2000.

[13] Nguyen, K., "Active Control of Helicopter Blade Stall," AIAA Paper 96-1221, AIAA Dynamics Specialists Conference, Salt Lake City, Utah, April 1996.

[14] Petot, D., "Differential Equation Modeling of Dynamic Stall," La Recherche Aérospatiale, Vol. 5, 1989, pp. 59-71.

[15] Truong, V. K., "2D Stall Model Based on a Hopf Bifurcation," Paper No. C23, Nineteenth European Rotorcraft Forum, Cernobbio, Italy, 1993.

[16] Leishman, J. G. and Beddoes, T. S., "A SemiEmpirical Model for Dynamic Stall," Journal of the American Helicopter Society, No. 4, No. 4, 1989, pp. 3-17.

[17] Fulton, M. and Ormiston, R. A., "Small-Scale Rotor Experiments with On-Blade Elevons to Reduce Blade Vibratory Loads in Forward Flight," Proceedings of the 54th Annual Forum of the American Helicopter Society, Washington, D. C., May 1998, pp. 433-451.

[18] De Terlizzi, M., "Blade Vortex Interaction and its Alleviation Using Passive and Active Control Approaches," Ph.D. Dissertation, University of California, Los Angeles, June 1999.

[19] Johnson, W., A Comprehensive Analytical Model of Rotorcraft Aerodynamics and Dynamics, Vol. I: Theory Manual, Palo Alto, CA, Johnson Aeronautics, 1988.

[20] Truong, V. K., "Prediction of Helicopter Rotor Airloads Based on Physical Modeling of 3D Unsteady Aerodynamics," Paper No. 96, TwentySecond European Rotorcraft Forum, Brighton, UK, September 1996.
[21] Peters, D. A., "Toward a Unified Lift Model for Use in Rotor Blade Stability Analyses," Proceedings of the 40th Forum of the American Helicopter Society, Arlington, Va., May 1984.

[22] Petot D.,, Arnaud, G., Harrison, R., Stevens, J., Diederich, O., van der Wall, B. G., Young, C., and Széchényi, E. "Stall Effects and Blade Torsion - An Evaluation of Predictive Tools," Journal of the American Helicopter Society, Vol. 3, 1999, pp. 320-331.

[23] Powell, M. J. D., "Unconstrained Optimization by Quadratic Approximation," Cambridge University, CP DAMTP 2000/NA14.

[24] Peters, D. A., Bayly, P. and Li, S., "A Hybrid Periodic-Shooting, Autopilot Method for Rotorcraft Trim Analysis," Proceedings of the 52th Forum of the American Helicopter Society, Washington, D.C., June 1996, pp. 780-792.

[25] Johnson, W., "Self-Tuning Regulators for Multicyclic Control of Helicopter Vibration," NASA, TP 1996, 1982.

[26] Meerkov, S. M., "Deceleration in the Search for the Global Extremum of a Function," $A v$ tomatika $i$ Telemekhanika, No. 12, No. 12, 1972, pp. 129-139. (Translated from Russian)

[27] Cribbs, R. and Friedmann, P. P., "Actuator Saturation and its Influence on Vibration Reduction by Actively Controlled Flaps," AIAA Paper 2001-1467, Proceedings of the 42th AIAA/ASME/ASCE/AHS/ACS Structures, Structural Dynamics and Materials Conference, Seattle, Wash., April 2001. 


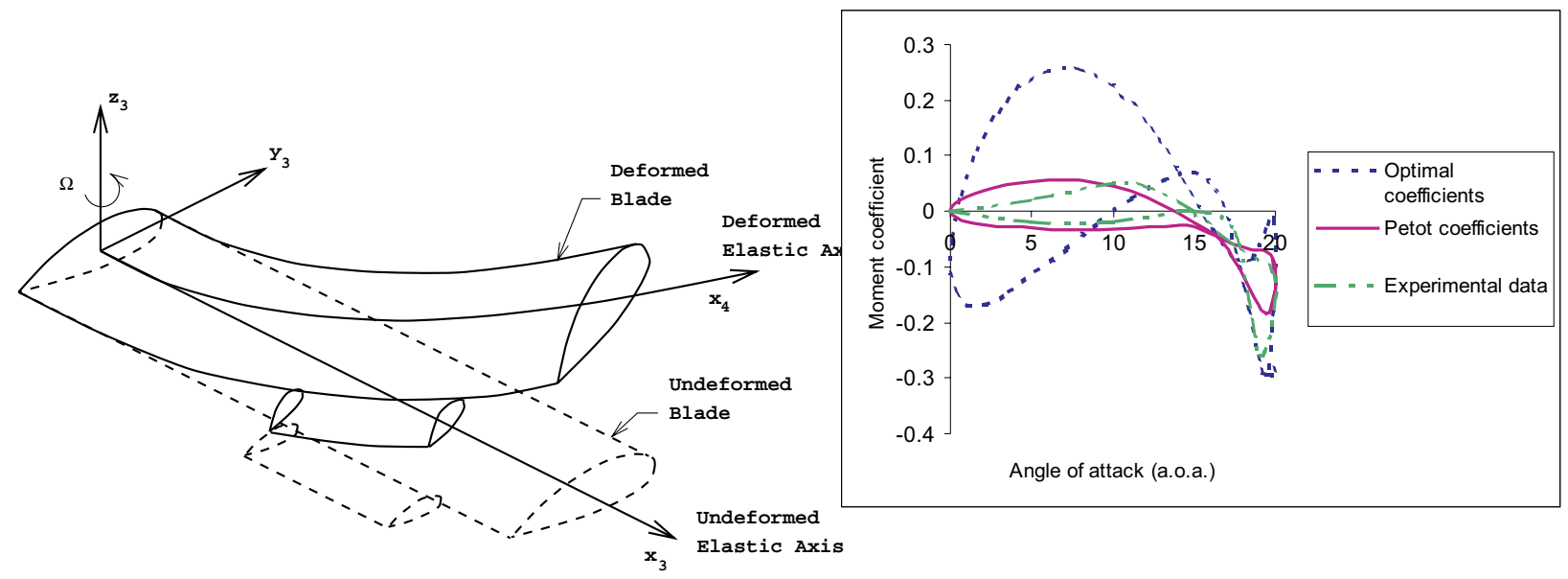

Figure 1: Schematic representation of the undeformed and deformed blade/actively controlled flap configurations.

Figure 3: Dynamic stall loads with two sets of empirical coefficients.
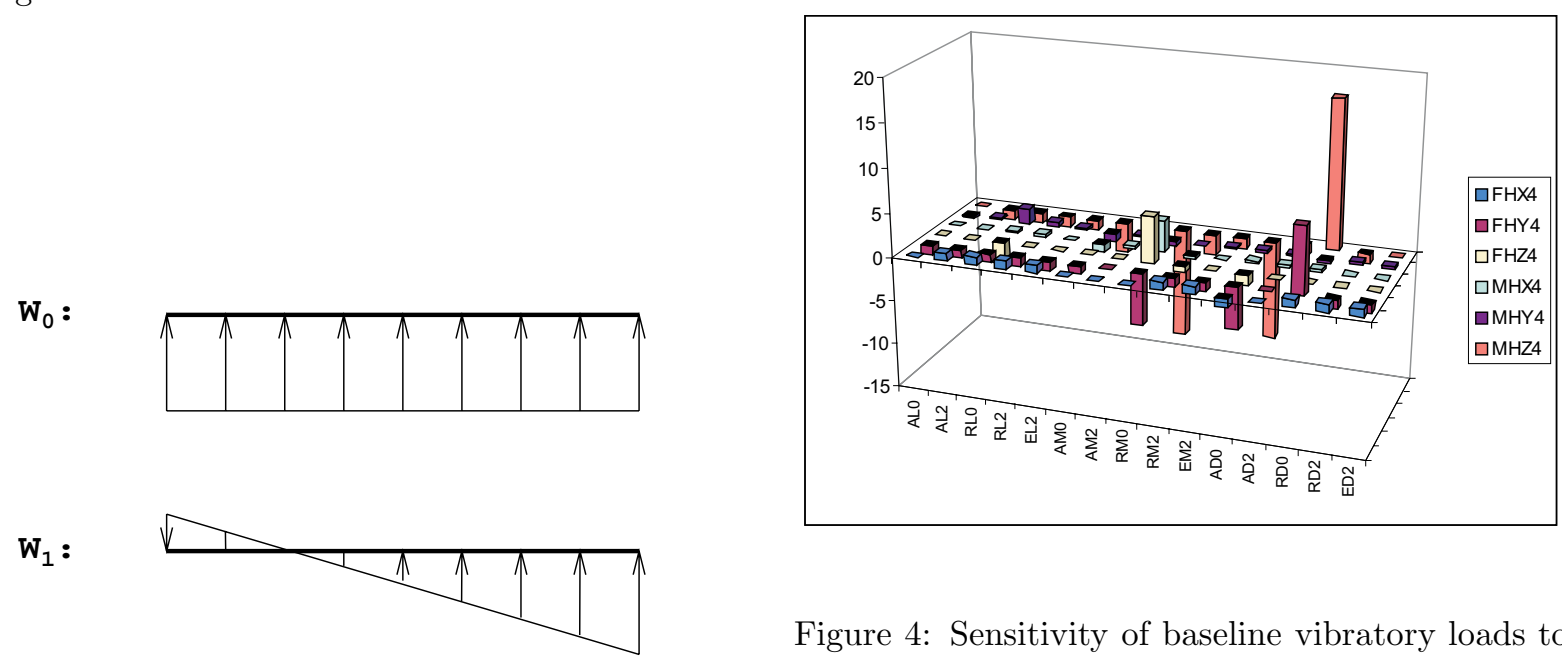

Figure 4: Sensitivity of baseline vibratory loads to dynamic stall empirical coefficients, $\mu=0.30$.

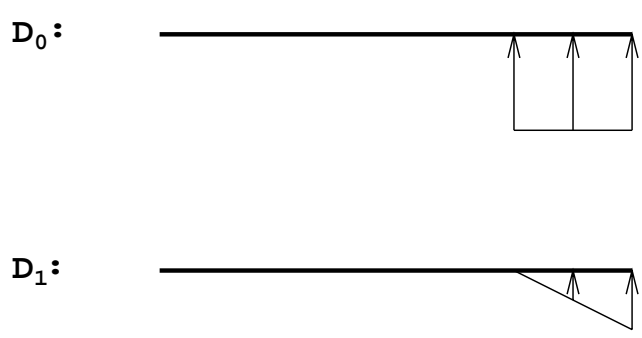

Figure 2: Normal velocity distributions corresponding to generalized airfoil and flap motions $W_{0}, W_{1}$, $D_{0}$, and $D_{1}$.

(a) single-flap configuration

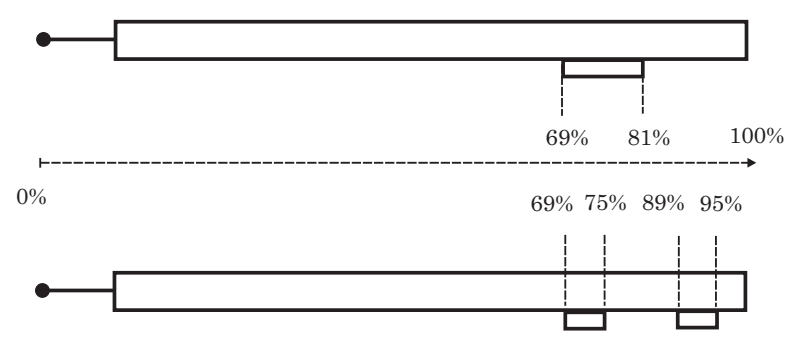

(b) dual-flap configuration

Figure 5: Single and dual flap configurations. 


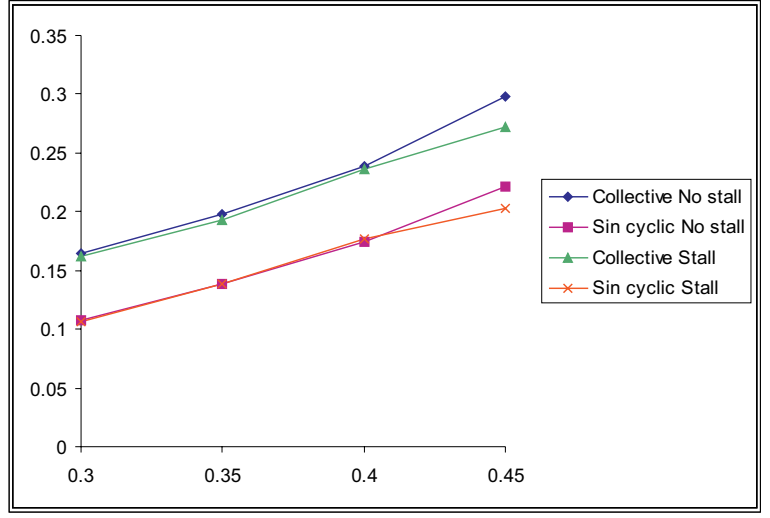

Figure 6: Influence of dynamic stall on collective and sine cyclic pitch.

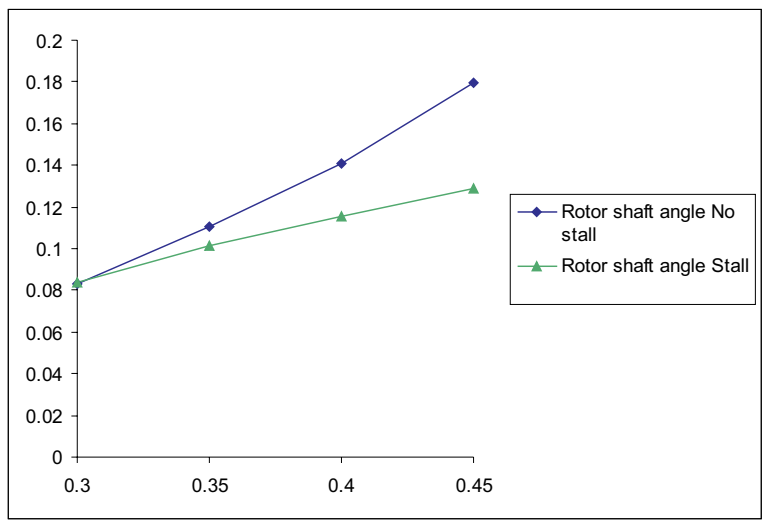

Figure 7: Influence of dynamic stall on rotor shaft angle.

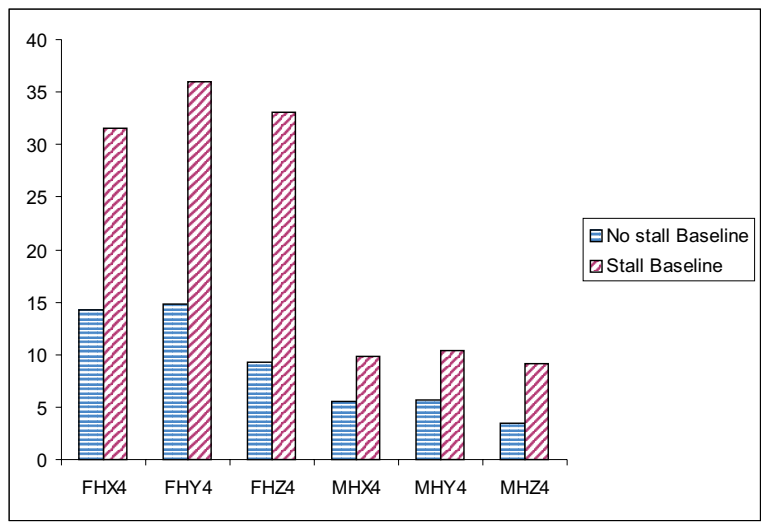

Figure 8: Influence of stall on baseline vibratory loads, $\mu=0.35$.

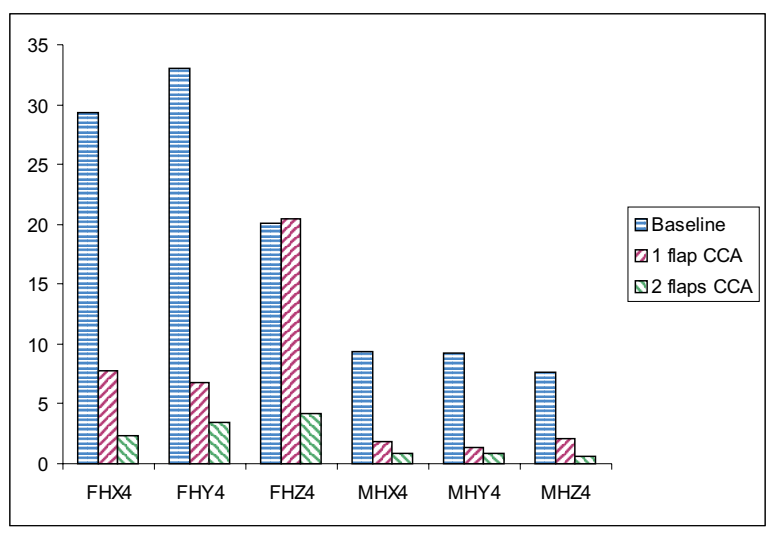

Figure 9: Vibration reduction, CCA, $\mu=0.35$.

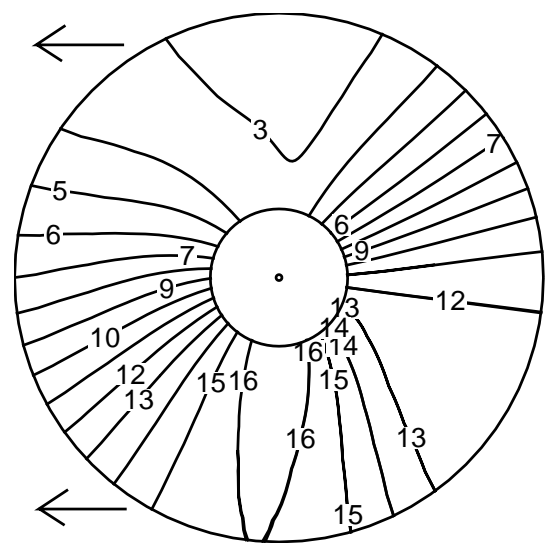

Figure 10: Angle of attack map in presence of dynamic stall, no control, $\mu=0.30$. The center of the figure represents the hub region, the outer circle depicts the rotor disk and the arrows show the direction of forward flight. Aerodynamic loads are neglected in the inner circle. 


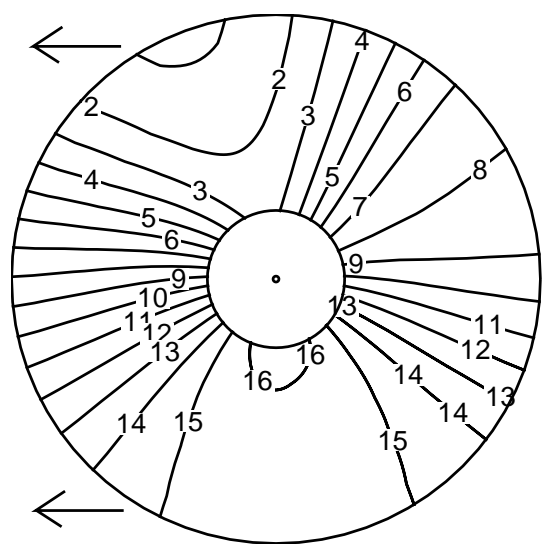

Figure 11: Angle of attack map in presence of dynamic stall, control, $\mu=0.30$. The center of the figure represents the hub region, the outer circle depicts the rotor disk and the arrows show the direction of forward flight. Aerodynamic loads are neglected in the inner circle.

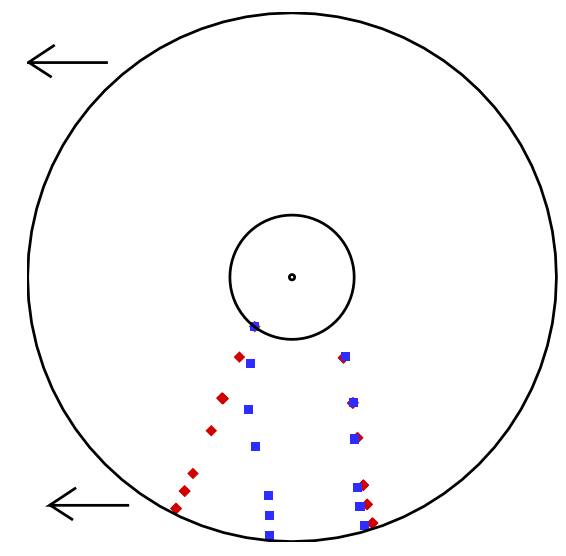

Figure 12: Dynamic stall locus control (squares) and no control (diamonds), $\mu=0.30$. The center of the figure represents the hub region, the outer circle depicts the rotor disk and the arrows show the direction of forward flight. Aerodynamic loads are neglected in the inner circle.

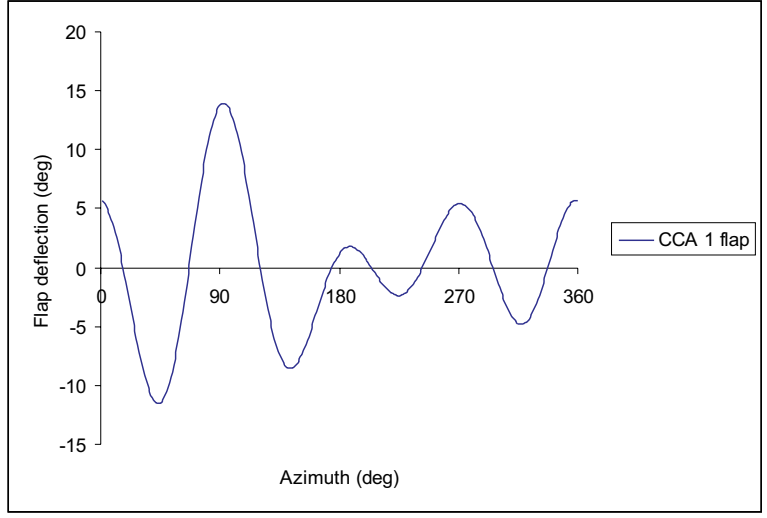

Figure 13: Flap deflections, CCA, single flap configuration, $\mu=0.35$.

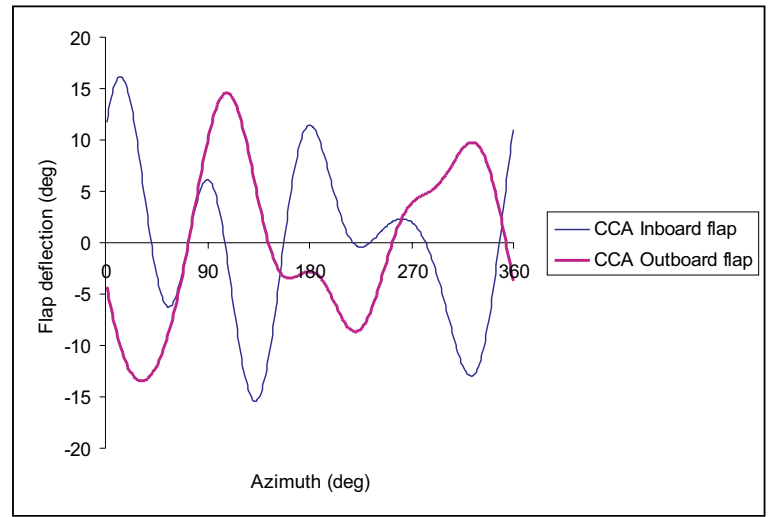

Figure 14: Flap deflections for dual flap configuration, CCA, $\mu=0.35$.

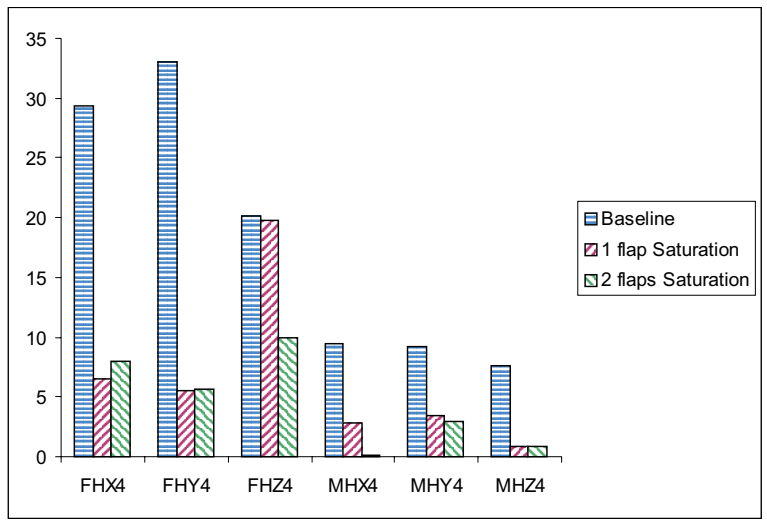

Figure 15: Vibration reduction with saturation limits, $\mu=0.35$. 


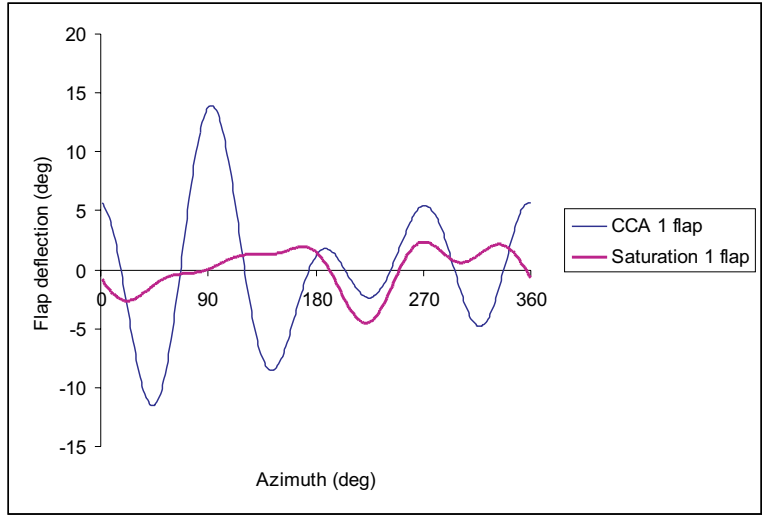

Figure 16: Flap deflections, effect of saturation, $\mu=0.35$.

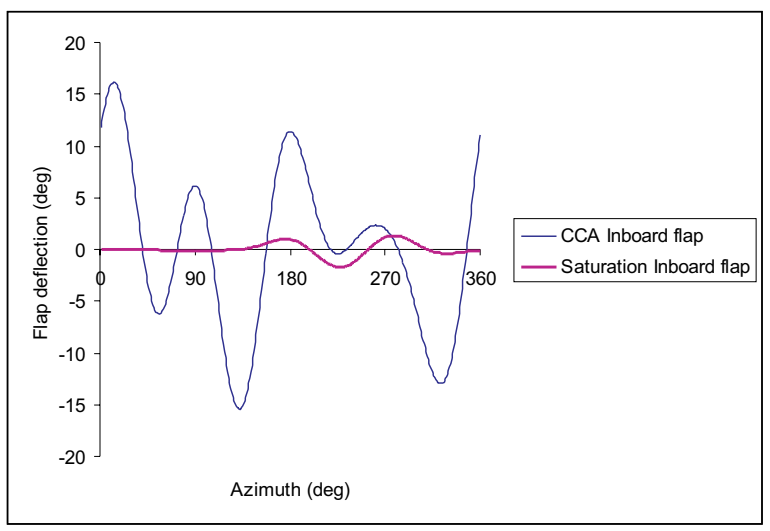

Figure 17: Inboard flap deflections, dual flap with saturation limits, $\mu=0.35$.

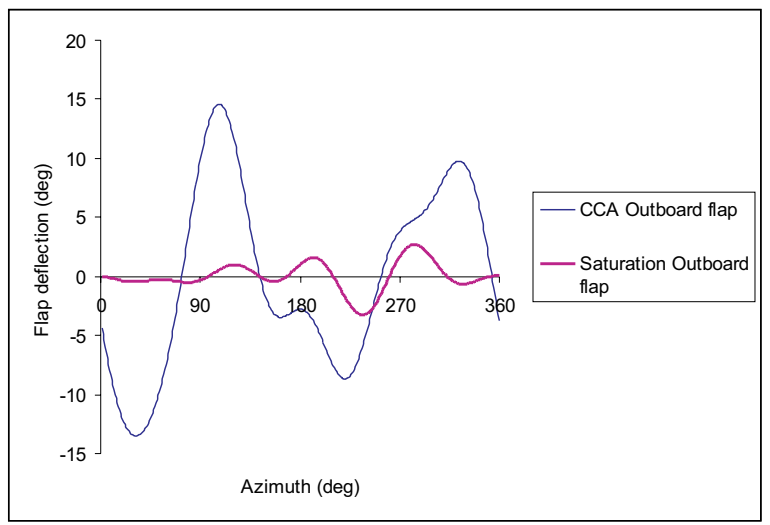

Figure 18: Outboard flap deflections, effect of saturation limits, $\mu=0.35$.

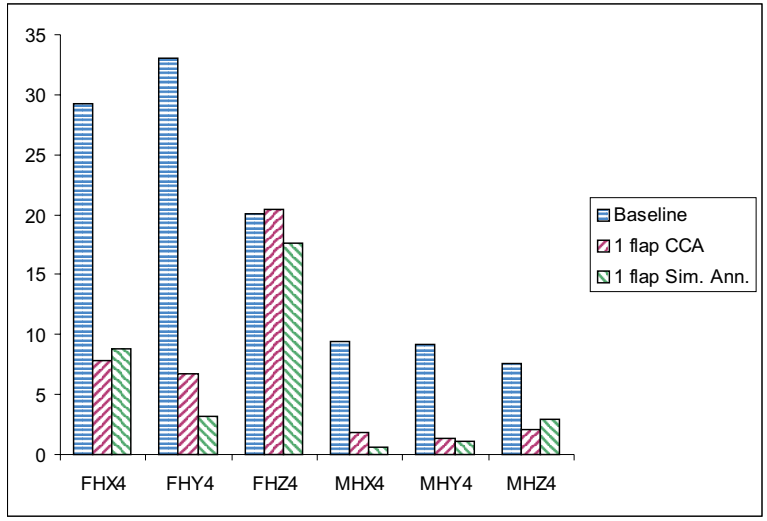

Figure 19: Vibration reduction, with SAA algorithm, $\mu=0.35$.

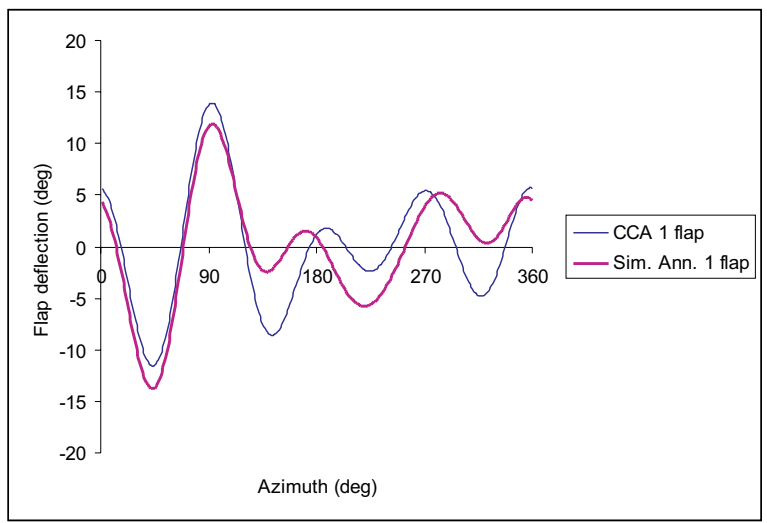

Figure 20: Flap deflections, SAA algorithm, $\mu=0.35$.

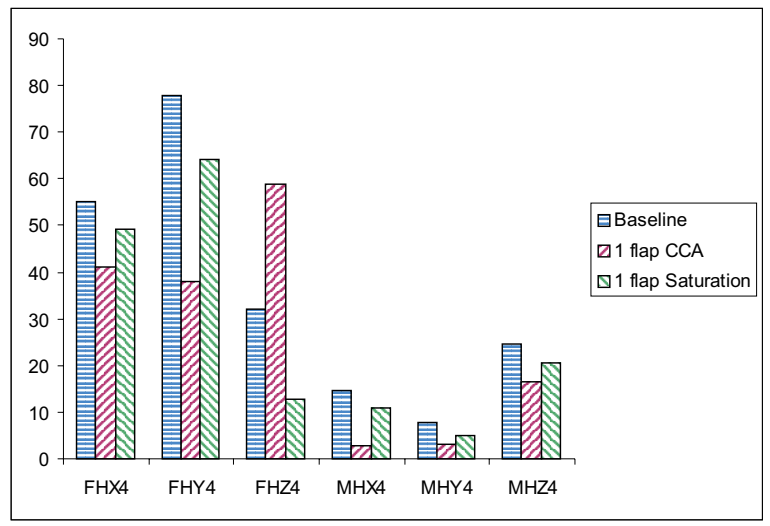

Figure 21: Vibration reduction, single flap, effect of saturation, $\mu=0.45$.

14

American Institute of Aeronautics and Astronautics 